\title{
Efficient ring perception for the Chemistry Development Kit
}

\author{
John W May ${ }^{*}$ and Christoph Steinbeck
}

\begin{abstract}
Background: The Chemistry Development Kit (CDK) is an open source Java library for manipulating and processing chemical information. A key aspect in handling chemical structures is the determination of the chemical rings. The rings of a structure are used areas including descriptors, stereochemistry, similarity, screening and atom typing. The CDK includes multiple algorithms for determining the rings of a structure on demand. Non-unique descriptions of rings were often used due to the slower performance of the unique alternatives.

Results: Efficient algorithms for handling chemical ring perception have been implemented and optimised in the CDK. The algorithms provide much faster computation of new and existing types of rings. Several optimisation and implementation considerations are discussed which improve real case usage. The performance is measured on several publicly available data sets and in several cases the new implementations were found to be more than an order of magnitude faster.

Conclusions: Algorithmic improvements allow handling of much larger datasets in reasonable time. Faster computation allows more appropriate rings to be utilised in procedures such as aromaticity. Several areas that require ring perception have also seen a noticeable improvement. The time taken to compute the unique rings is now comparable allowing a correct usage throughout the toolkit. All source code is open source and freely available.
\end{abstract}

Keywords: Rings, Cycles, CDK

\section{Background}

The Chemistry Development Kit (CDK) [1,2] is an open source Java library for manipulating chemical information. A key aspect of manipulating and querying chemical information is the ability to define and reason about attributes of chemical structures. Describing the rings in a structure is fundamental and a prerequisite of other attributes.

There is often a disconnect between how chemical rings are numbered and what is useful for computation. Conflicting definitions of rings contribute towards discrepancies between chemistry toolkits such as assigning aromaticity. The CDK does not provide a single strict definition of what rings are present in a structure. The ring information is considered auxiliary with different

\footnotetext{
*Correspondence: johnmay@ebi.ac.uk

Cheminformatics and Metabolism, European Molecular Biology Laboratory-European Bioinformatics Institute (EMBL-EBI), Wellcome Trust Genome Campus, Hinxton, Cambridge, UK
}

algorithms utilised for a specific use-case. Some considerations of the differences will be touched upon but a thorough review is provided by [3,4] and [5].

There are several key properties we wish to know: is an atom or bond in a ring, what size is the ring and what are the other atoms and bonds in the ring? This information can be stored as an attribute of each atom or bond, as a collection of rings on the structure or computed on demand. With the provision of multiple algorithms it is undesirable to store all the information but invariant properties including membership and smallest ring size could be stored as an attribute of an atom or bond.

The ring properties can be used in many procedures throughout the library. In similarity searching and screening the creation of chemical fingerprints [6] may include ring size or membership to reduce the number of false positives. When matching atoms and bonds between structures the ring properties can be used in early elimination of infeasible matches or to disfavour ring opening and closing. Ring properties are also utilised in structure 
patterns (SMARTS [7]) where ring membership, size and number of rings can be queried.

It is essential that different structure resonance forms are treated as equivalent, one approach is to treat bonds in aromatic ring systems as delocalised. Conversely a delocalised structure may have been provided without specified bond orders. The ring properties can be used to localise and delocalise the bonds between aromatic and Kekulé representations.

Geometric isomers (double-bond stereochemistry) should not be encoded when the bond is involved in a rigid ring. Rigidity is approximated by only allowing stereoconfigurations in rings with more than seven atoms. Groups of interdependent stereocenters can be identified by recursively checking the rings in a structure [8].

Improving the core ring perception algorithms can influence many areas and it is important that efficient algorithms are used.

\section{Graph theory preliminaries}

Although more comprehensive and accurate methods exist, chemical structures can be represented and efficiently modelled as graphs [9]. The algorithms used for ring perception are not specific to chemical structures and require several formal definitions. The basic concepts for these are briefly introduced here. A graph is composed of a set of vertices $V$ and a set of edges $E$. Each vertex or edge may be labelled with a value. Two vertices are adjacent if an edge exists which contains the two vertices. The vertices of an edge are known as the endpoints, each endpoint is said to be incident to the edge. A degree of a vertex is the number of incident edges. If the endpoints are unordered, an edge is said to be undirected. Simple graphs have no edges connecting the same vertex (loops) and no edges which share the same endpoints (multiedges). We model a chemical structure as simple undirected labelled graph where the atoms and bonds are labels on the vertices and edges. Although the edges have a numeric value (bond order) they are not treated as weighted.

A walk is a sequence of vertices and edges connecting two vertices. If the start and end of the walk are the same, the walk is closed. Otherwise the walk is open. A walk is simple if it contains no repeated edges and elementary if there are no repeated vertices. A simple walk that is also open it is referred to as a path. Two vertices are connected if there is a path between them. A graph is connected if each vertex can be reached from every other vertex. A connected component (Conn $\operatorname{Comp}(G))$ in an undirected graph is a subgraph in which every vertex is connected.

A cycle is a closed walk. Graphs containing a cycle are said to be cyclic or acyclic if no cycle is present. Acyclic simple graphs are referred to as a tree. A ring in a chemical structure is best described as an elementary cycle. The cycle has no repeating vertices or edges and each vertex has a degree of 2 (in the cycle). This definition includes envelope rings of structures like napthalene and azulene. As we are primarily concerned with chemical structures herein we use the term cycle to refer to elementary cycle.

A cycle basis is a set of cycles which can be used to generate all other cycles (cycle space) of the graph. Representing a cycle as a set of edges, a new cycle can be generated using the symmetric difference (XOR, $\oplus$-summing) of the edge sets of two cycles whose edge sets intersect. A minimum cycle basis is a cycle basis of minimum weight, in an unweighted graph the weight is simply the number of edges. When there is more than one basis with the same weight the choice between them is arbitrary as either can be used to generate the cycle space.

\section{Cycle membership}

The first step in cycle processing for a chemical structure is to efficiently determine which vertices and edges of the graph belong to a cycle. In PubChem-Compound [10] (Aug 2013) $97.3 \%$ of structures $(47,745,887)$ contained a cycle. Although the proportion of structures containing a cycle is high only $59.3 \%$ of the heavy atoms and $57.3 \%$ of bonds were cyclic. Eliminating these acyclic vertices and edges from further processing reduces the size of the computation.

The SpanningTree was introduced in the CDK to eliminate acyclic vertices and edges, reducing the runtime of existing algorithms [11]. A graph $H$ is a subgraph of a graph $G$ if the vertices $V$ and edges $E$ of $H$ are a subset of $G$. A subgraph $G$ is said to be a spanning subgraph of $H$ if every vertex of $H$ is present in $G$. The edges in chemical structures are unweighted and so the minimum spanning tree is a tree with the smallest number of edges. Given an input structure a spanning tree is created which contains a subset of the edges that span the vertices but contains no cycles. The SpanningTree class uses a greedy algorithm [12] to sequentially build up this tree. Cyclic vertices and edges are determined by finding a path in the tree between the two endpoints of an edge which was not included. Any edge that is not in the spanning tree is cyclic and any path in the tree which connects the two endpoints contains vertices and edges that are also cyclic. The number of paths to find depends on the number of edges not included in the spanning tree. Structures containing a large number of rings will have more edges removed and more paths to find. Discovery of a path in the tree is implemented as depth-first-search and the entire tree may be traversed for each removed edge.

\section{Cycle sets}

In addition to determining if a vertex or edge is cyclic, one would also like to know the sizes of cycles and the walks. 


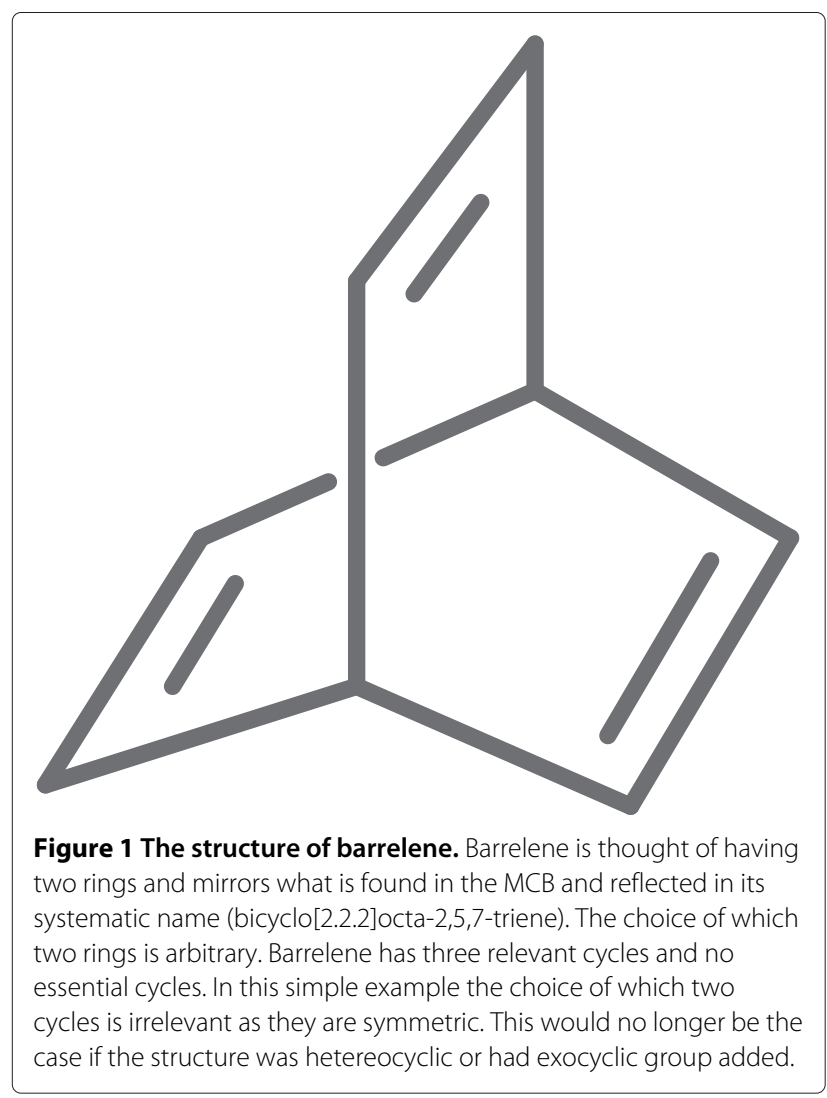

There is an exponential number of elementary cycles in a graph and smaller subsets of this have subsequently been defined and used in various aspects of chemical information processing.

\section{Smallest set of smallest rings/minimum cycle basis}

A well known set of cycles is the Smallest Set of Smallest Rings (SSSR). The SSSR was originally defined as a minimum length Kirchhoff-fundamental basis but has evolved to refer to a minimum cycle basis (MCB). The original definition of SSSR does not always contain the shortest cycles and was computationally intractable [3]. To avoid confusion the term SSSR will now only be used in reference to CDK implementation names. As introduced previously the MCB is a polynomial set of cycles which can be used to generate the cycle space. As the MCB may not be unique it has little direct use in similarity, aromaticity, depiction or other descriptive features. It is also not required to find the shortest cycle through each edge or vertex which can be accomplished without checking the cycles form a basis. Although the MCB is not unique, the number of cycles it contains is. This value is the circuit rank ${ }^{\mathrm{a}}$ and is the number of edges that would need to be removed to make the graph acyclic (a spanning tree). For these reasons the size of the MCB agrees with de-facto standards and chemical nomenclature (Figure 1). The formula $|E|-$ $|V|+|\operatorname{Conn} \operatorname{Comp}(G)|$ provides the circuit rank without computation of the cycle walks [5].

The original algorithm [13] utilised in the CDK was shown to be incorrect and can not guarantee completion on all graphs [3]. Although one may consider such cases rare in four of the five tested chemical data sets (Table 1) at least one structure was found which caused the CDK implementation to halt indefinitely. The algorithm is still partially used in other cheminformatics libraries [14]. The implementation was replaced with a correct algorithm [3] (SSSRFinder) which also provides uniquely defined cycle sets as alternatives to the MCB.

In general the CDK library has been relying less on $\mathrm{MCB}$ as it has little use beyond counting the number of rings and generating the cycle space. Both of these tasks can be achieved more efficiently with other procedures. The implementations provided in the CDK are primarily for reference and their use in computing other uniquely defined cycle sets.

\section{Essential and relevant cycles}

The essential and relevant cycles are a uniquely defined set of cycles. The MCB is non-unique when there are multiple minimum cycle bases and an arbitrary choice of a single basis can generate the cycle space. The essential cycles is the intersect of these minimum cycle bases whilst the relevant cycles is the union. When a graph has a single unique MCB it is equal to both the essential and relevant cycles. As a subset of the MCB the essential cycles do

Table 1 Chemical structure sets used to measure performance

\begin{tabular}{llll}
\hline Identifier & n structures & Description & Available \\
\hline chebi_108 & 26,790 & ChEBI Release 108 [15] & www.ebi.ac.uk/chebi \\
nci_aug00 & 250,172 & NCl Aug 2000 [16] & cactus.nci.nih.gov/download/nci \\
zinc_frag & 504,074 & Zinc Clean Fragments & zinc.docking.org/subsets/clean-fragments \\
& & Ph7 2013-04-12 [17] & Www.ebi.ac.uk/chembl \\
chemb1_17 & $1,318,180$ & ChEMBL Release 17 [18] & zinc.docking.org/subsets/clean-leads \\
zinc_leads & $5,135,179$ & Zinc Clean Leads Ph7 2013-05-31 [17] &
\end{tabular}

The number of structures is the number which were successfully read from SMILES [19] notation. The ChEBI and ChEMBL datasets had a small number erroneous SMILES string which could not be interpreted. 
not form a basis and cannot be used to generate the cycle space. Like the MCB the essential cycles are always polynomial in number. Counter-intuitively, structures such as barrelene (Figure 1) contain no essential cycles. The relevant cycles do form a basis but may be exponential in number.

The uniqueness of these cycle sets make them desirable for describing chemical entities. The essential cycles have been utilised in the CDK for similarity searching techniques including generation of fingerprints and for the structure query patterns. Unfortunately the computation of the unique essential and relevant cycles (using the SSSRFinder) takes much longer than the non-unique $\mathrm{MCB}$. The increased computation runtime has generally meant the MCB has been favoured.

\section{All elementary cycles}

When considering all cycles, the number of cycles can be very large and infeasible to compute for fullerene-like and cyclophane-like structures. The set of all cycles can be generated using a cycle basis or computed directly [20]. Direct computation is more efficient and is provided in the CDK as the AllRingsFinder. One major drawback of the existing implementation is the dependence on a time measure to determine feasibility. The time was measured from when the algorithm started and aborted if the elapsed time exceeded a set threshold. Whether the algorithm completes then depends on the machine specification and also the current load on the processor. The timeout was also generally left at a value too high (5 seconds) for larger datasets. To demonstrate this, the timeout threshold was varied and tested on a small dataset. The number of structures that the algorithm successfully completed was measured. Increasing the threshold to longer than a second provides only a small gain in coverage (Figure 2). A timeout of just $50 \mathrm{~ms}$ allowed $99.4 \%$ of the structures to complete in 32 seconds. Leaving the timeout at the default value of $5000 \mathrm{~ms}$ allowed $99.8 \%$ of structures to complete but took nearly 10 times longer (291 seconds) (Figure 3 ). This could be an artefact of hardware improvements but highlights the difficulties in choosing an appropriate value when using a timeout. The set of all cycles was used throughout the library in fingerprint generation, similarity searching [21], descriptors, kekulisation and fragmentation. The cycles were also partially utilised in aromaticity perception.

\section{Implementations}

The processing of cycles in the CDK has been streamlined and optimised. Improved algorithms for determining cycle membership and the uniquely defined essential and relevant cycles have been implemented in the CDK library. The algorithms are split across several classes

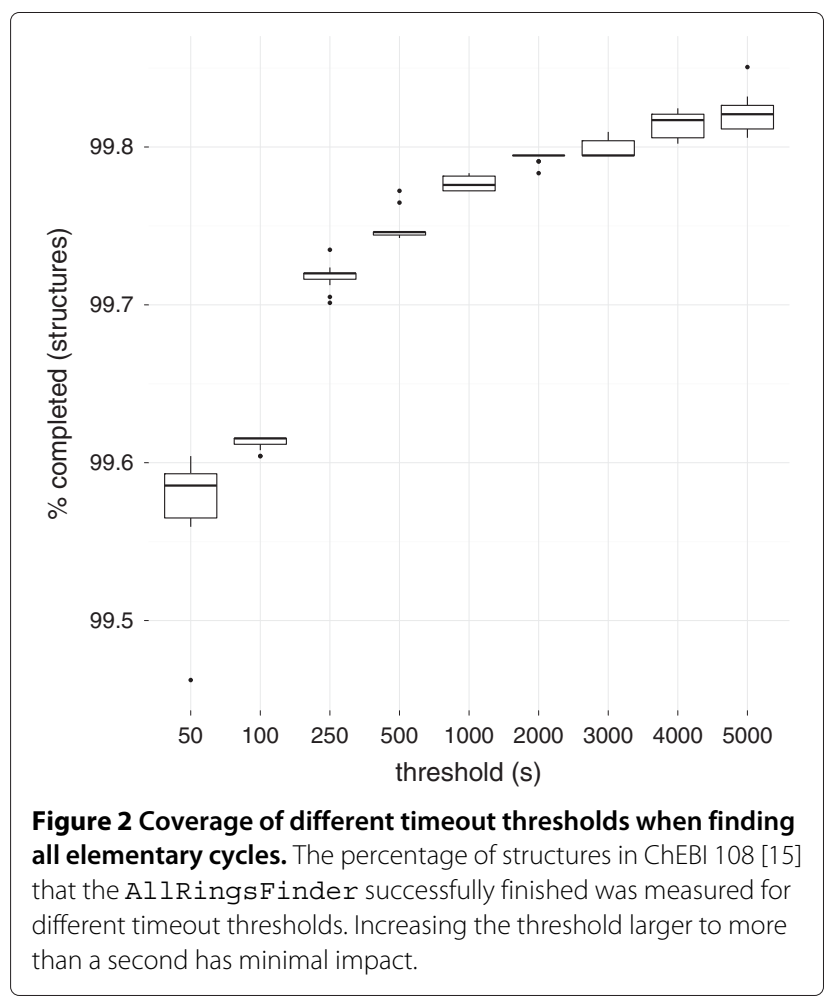

allowing an expert user to pick and choose. For simplicity a facade, Cycles (Figure 4), provides generation of the cycle sets described and applies preprocessing optimisations. Specific implementation details are discussed and measured in the results.

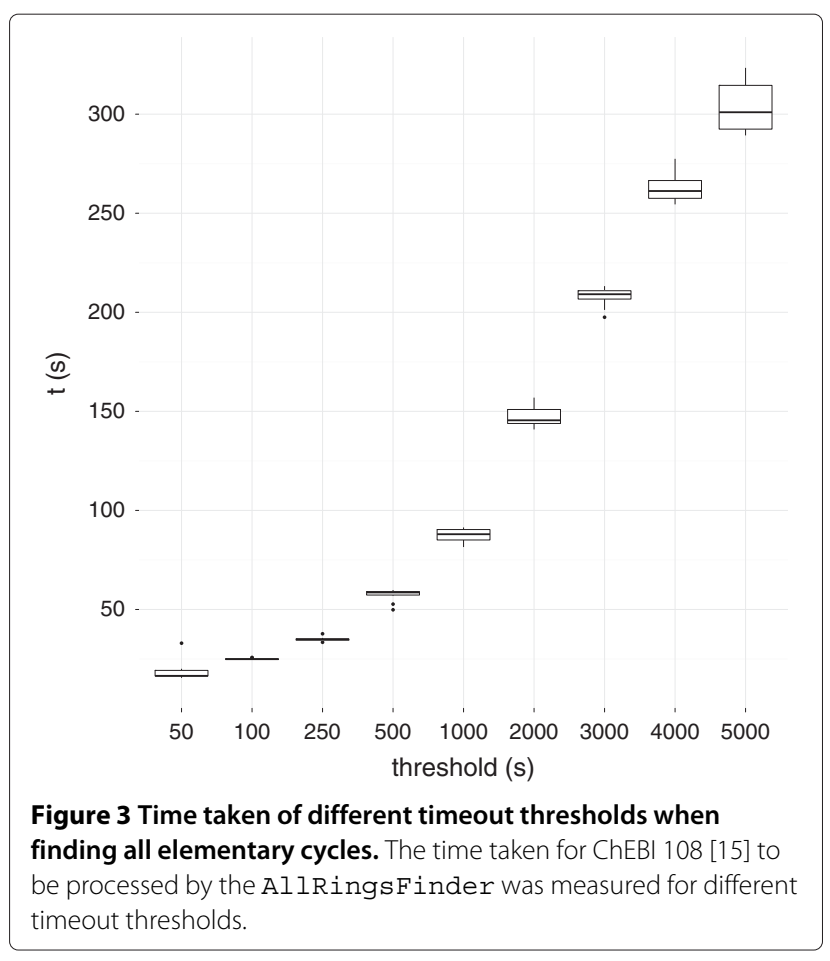




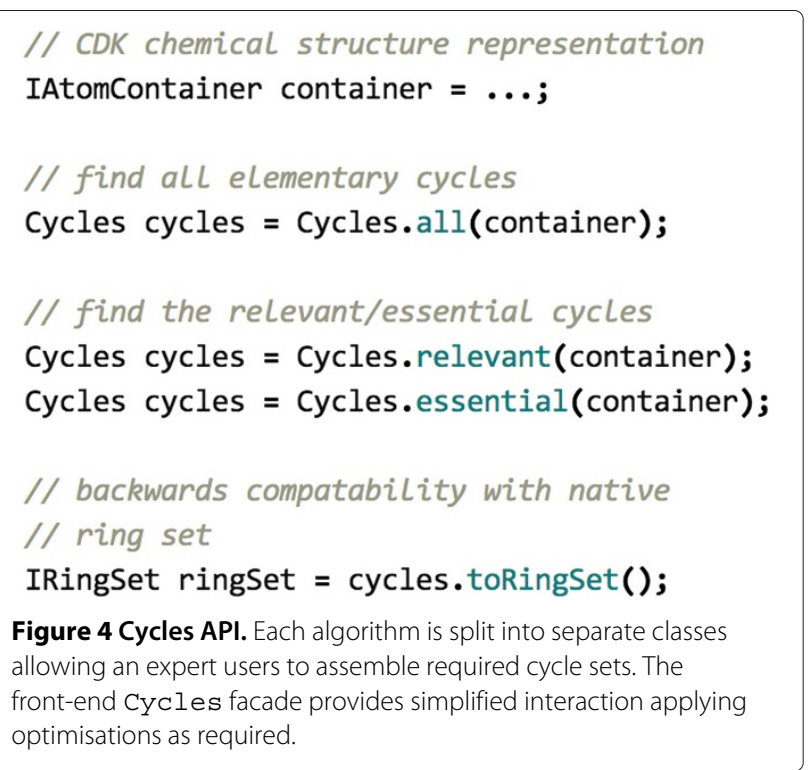

\section{Graph representations}

A graph can be represented and stored using several data structures [22] (Figure 5). The choice of data structure can dramatically affect performance. A coordinate or edge-list representation stores the vertices and edges as separate lists. The edge list is memory efficient but inefficient to determining adjacency where every edge must be checked. An adjacency matrix is a square matrix with a boolean value indicating whether two vertices are adjacent. Matrix representations offer constant time adjacency checking but require every vertex to be checked in order to obtain a list of neighbours or degree. The matrix representation is less memory efficient and requires quadratic space to store. In an adjacency/incidence list each vertex stores adjacent vertices or incident edges. Testing adjacency is bounded by the number of adjacent vertices, the degree [22]. The degree and the set of adjacent vertices can be obtained in constant time.

The choice of data structure depends on properties being modelled, and which algorithms will be used. Chemical structures are generally small $(|V|<100)$ and each vertex is only adjacent to a few other vertices (sparse). Although more costly in memory and for modifications the attributes of chemical structures make the adjacency (or incidence) list representation preferable.

The CDK currently uses an edge list representation to store chemical structures. Conversion of the CDK native data type to an adjacency list is relatively quick but can become significant if carried out multiple times. Many of the existing algorithms used an optimised representation but benefit was seen by avoiding the slower CDK native objects and minimising reconversion. The overhead introduced for converting the CDK objects (Table 2) could be minimised by loading directly into

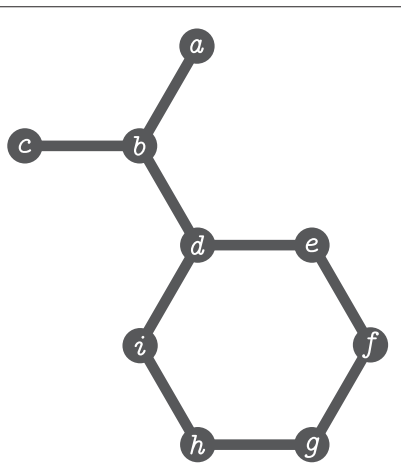

$$
\begin{gathered}
\{a, b\} \\
\{b, c\} \\
\{b, d\} \\
\{d, \quad e\} \\
\{d, \quad i\} \\
\{e, f\} \\
\{f, \quad g\} \\
\{g, \quad h\} \\
\{h, i\} \\
\text { edge list }
\end{gathered}
$$

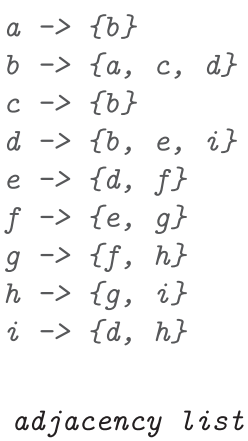

Figure $\mathbf{5}$ Graph representations. An abstract graph representation of a chemical structure (propan-2-ylbenzene) and different representations. The labelled vertices $(a, b, \ldots i)$ correspond to the values in the representations. 
an more optimal data structure. When comparing to existing methods the conversion time is included in comparisons.

\section{Results and discussion}

Here we describe the optimisations and measure the performance on several chemical datasets (Table 1). All measurements were performed on a $2.66 \mathrm{GHz}$ Intel Core i7 processor using Java version 1.7.0_21. The unprocessed benchmark results are provided as Additional files 1, 2 and 3.

\section{Cycle membership}

The existing algorithm used in SpanningTree was for graphs with weighted edges [12]. In an unweighted graph any spanning tree is the minimum spanning tree. A spanning tree in an undirected, unweighted graph can be constructed with a depth- or breath-first-search [23]. Although efficient in construction, the spanning tree still requires additional operations to determined the cyclic vertices and edge. A more efficient approach is to compute the biconnected (2-connected) components of the graph. The biconnected components can be found using a single depth-first search [24]. A vertex is biconnected if removing it from the graph does not increase the number components. A biconnected component is a maximal connected subgraph where every vertex is biconnected. In addition to detecting the cyclic vertices and edges the procedure also partitions the graph in to separate components which correspond to a separate ring systems in the chemical structure. If the number of edges is equal to the number of vertices, $|E|=|V|$, then the circuit rank is 1 and the component is an elementary cycle. Such components correspond to the isolated and spiro ring systems in a chemical structure whilst the other biconnected components are the fused and bridged ring systems. The simple elementary cycles need no further processing and can be skipped from the more computationally intensive algorithms.

To measure the impact by utilising the biconnected components the time taken to compute the MCB was

\begin{tabular}{|c|c|c|c|c|c|}
\hline \multirow[t]{2}{*}{ Chemical structure } & \multirow[t]{2}{*}{$n$ structures } & \multicolumn{2}{|c|}{ Adjacency list } & \multicolumn{2}{|c|}{ Incidence list } \\
\hline & & $t(\mathrm{~ms})$ & sdev & $t(\mathrm{~ms})$ & sdev \\
\hline chebi_108 & 26,790 & 167 & 14 & 238 & 15 \\
\hline nci_aug00 & 250,172 & 998 & 49 & 1,347 & 53 \\
\hline zinc_frag & 504,074 & 1,466 & 13 & 2,029 & 29 \\
\hline chembl_17 & $1,318,180$ & 8,308 & 33 & 11,977 & 246 \\
\hline zinc_leads & $5,135,179$ & 22,537 & 582 & 33,567 & 2368 \\
\hline
\end{tabular}

measured on the chemical structure sets. Although processing only the cyclic vertices and edges improves performance an even larger gain can be seen by processing only the non-simple biconnected separately (Figure 6). The largest performance improvement was seen for the zinc data sets that contain fewer fused ring systems.

The biconnected components were already used internally for other cycle computations (SSSRFinder). A new RingSearch utility was written with an algorithm optimised for small graphs using binary sets. The implementation provides logical testing of cycle membership for vertices and edges as well as partitioning the components and creating fragments of the input structure.

The time taken on several chemical datasets showed the new implementation performs well compared to the SpanningTree (Figure 7). The new algorithm can process between 100,000 and 300,000 structures per second where the majority of time (Table 3) was spent in the conversion (Table 2). The zinc_leads data has almost five times the number of structures than chembl_17 but the SpanningTree actually finished in less time (Table 3). This is because chembl_17 contains more fused-ring systems that cause a bottle neck in processing. The difference is emphasised by measuring the performance on single structures containing large fused ring systems (Table 4 ).

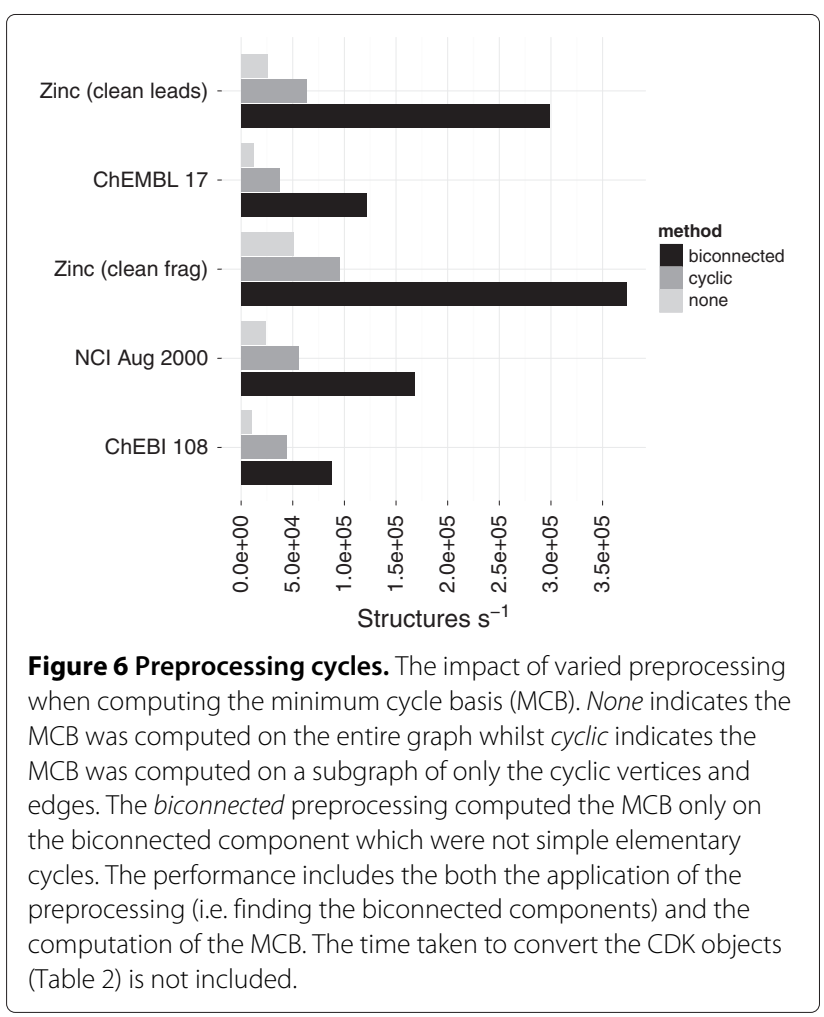




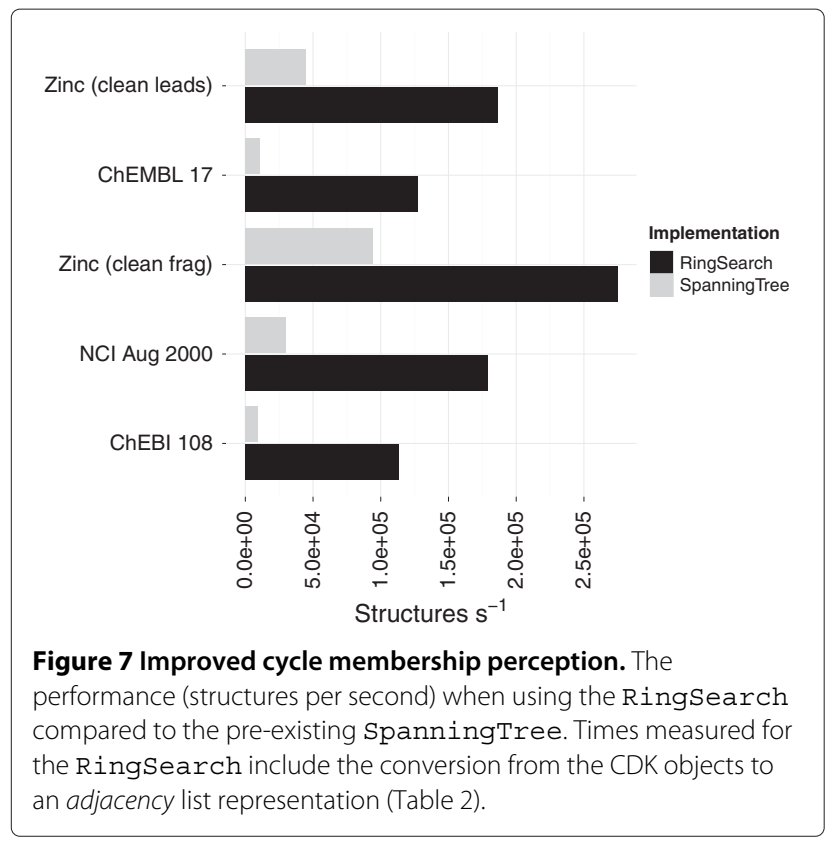

\section{Minimum cycle basis}

The new implementation MinimumCycleBasis computes the MCB as by-product of the relevant cycles [26]. Although this algorithm has a higher computational complexity than the existing (SSSRF inder) in practice it was found to be several factors faster (Figure 8). Although the existing implementation scales better, processing small graphs and an optimised implementation with fewer overheads can run faster.

The absolute time taken for processing structures from zinc_frag has dropped from $\sim 47$ to $\sim 2$ seconds whilst processing chembl_17 went from $\sim 4$ minutes to $\sim 15$ seconds (Table 5). The algorithm to compute the MCB and relevant cycles [26] first computes an initial set of cycles in a compact representation that is then reduced to either a MCB or the relevant cycles. Determining the

Table 3 Average and median $(n=15)$ time taken to determine ring membership

\begin{tabular}{|c|c|c|c|c|c|c|}
\hline \multirow{2}{*}{ Chemical structure } & \multicolumn{3}{|c|}{ SpanningTree } & \multicolumn{3}{|c|}{ RingSearch } \\
\hline & $\begin{array}{l}\text { mean } \\
t(\mathrm{~ms})\end{array}$ & $\begin{array}{l}\text { median } \\
t \text { (ms) }\end{array}$ & sdev & $\begin{array}{l}\text { mean } \\
t(\mathrm{~ms})\end{array}$ & $\begin{array}{l}\text { median } \\
t(\mathrm{~ms})\end{array}$ & sdev \\
\hline chebi_108 & 2,969 & 2,826 & 246 & 236 & 212 & 90 \\
\hline nci_aug00 & 8,440 & 8,451 & 59 & 1,396 & 1,372 & 88 \\
\hline zinc_frag & 5,338 & 5,353 & 77 & 1,833 & 1,818 & 60 \\
\hline chembl_17 & 122,357 & 122,493 & 616 & 10,325 & 10,303 & 98 \\
\hline zinc_leads & 114,710 & 115,067 & 837 & 27,496 & 27,502 & 215 \\
\hline
\end{tabular}

Times exclude 10 but include conversion. It should be noted that the majority of the time spent in Ringsearch was for the conversion (adjacency). Unprocessed measurements are provided in Additional file 1: Cycle membership benchmark.
Table 4 Average $(n=50)$ time taken to determine ring membership in several complex structures from $\mathrm{ChEBI}$ and FULLERENE [25]

\begin{tabular}{lllll}
\hline & \multicolumn{2}{c}{ SpanningTree } & RingSearch \\
\cline { 2 - 5 } Chemical structure & $\boldsymbol{t}(\mathrm{ms})$ & sdev & $\boldsymbol{t}(\mathrm{ms}) \mathrm{sdev}$ \\
\hline cubane (CHEBI:33014) & 1.18 & 1.24 & 0.21 & 0.66 \\
dodecaboride (CHEBI:51706) & 1.11 & 0.80 & 0.05 & 0.01 \\
octacontaboron (CHEBI:50252) & 100.18 & 52.87 & 0.44 & 0.18 \\
$\mathrm{C}_{60}$ fullerene (CHEBI:33128) & 11.15 & 2.92 & 0.30 & 0.03 \\
$\mathrm{C}_{70}$ fullerene (CHEBI:33195) & 10.44 & 4.25 & 0.88 & 0.23 \\
$\mathrm{C}_{320}$ fullerene (FULLERENE) & 423.06 & 166.30 & 0.61 & 0.32 \\
$\mathrm{C}_{720}$ fullerene (FULLERENE) & 3100.71 & 1171.62 & 1.65 & 0.66 \\
\hline
\end{tabular}

initial set of cycles uses a shortest paths procedure where an ordering is imposed on vertices [27]. With the vertices ordered by degree if the biconnected component is known to be a non-simple component (i.e. fused or bridged) then only shortest path searches from vertices with deg $>2$ will yield new cycles to add to the initial cycles. In practice this reduces the number of shortest path searches, for example in the common naphthalene and anthracene substructures from 10 and 14 to 2 and 4 respectively.

The cycle basis is formed by incrementally adding candidate cycles of increasing size. In this case the candidates are the initial set of cycles [26]. A candidate is added to the

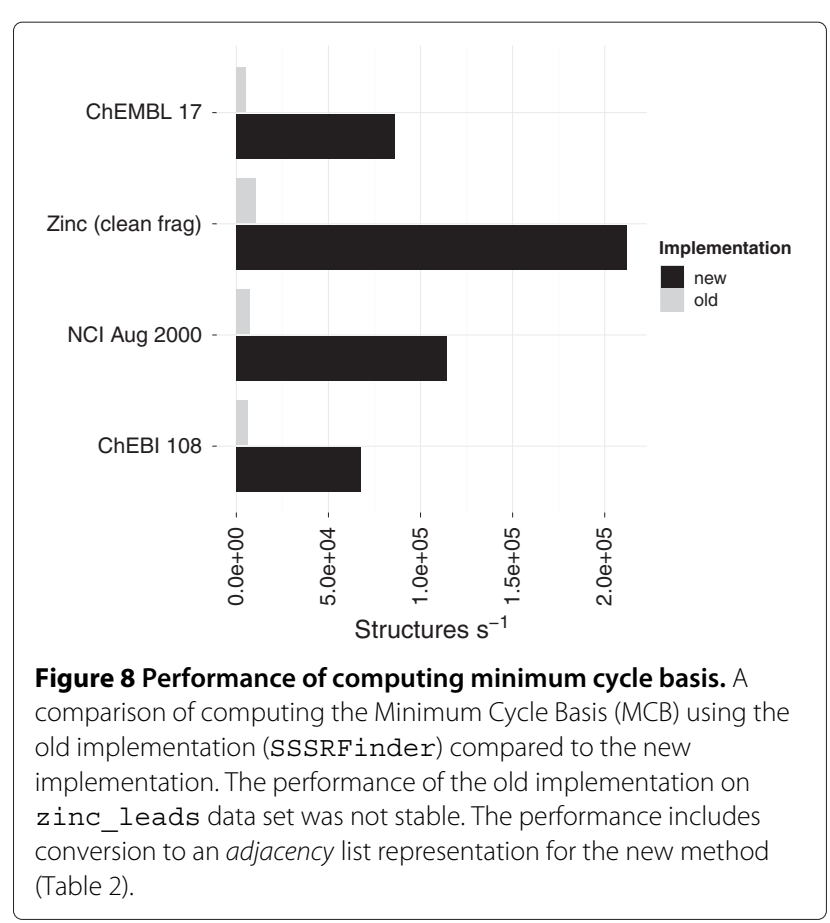


Table 5 Average and median $(n=15)$ time taken to compute the minimum cycle basis (MCB) using the existing and improved implementations

\begin{tabular}{|c|c|c|c|c|c|c|}
\hline \multirow{2}{*}{ Chemical structure } & \multicolumn{3}{|c|}{ MCB (old) } & \multicolumn{3}{|c|}{ MCB (new) } \\
\hline & $\begin{array}{l}\text { mean } \\
t(\mathrm{~ms})\end{array}$ & $\begin{array}{l}\text { median } \\
t(\mathrm{~ms})\end{array}$ & sdev & $\begin{array}{l}\text { mean } \\
t(\mathrm{~ms})\end{array}$ & $\begin{array}{l}\text { median } \\
t(\mathrm{~ms})\end{array}$ & sdev \\
\hline chebi_108 & 4,200 & 4,020 & 695 & 396 & 353 & 160 \\
\hline nci_aug00 & 34,762 & 34,330 & 1,685 & 2,193 & 2,125 & 211 \\
\hline zinc_frag & 47,752 & 47,844 & 1,982 & 2,376 & 2,330 & 153 \\
\hline chembl_17 & 245,620 & 245,592 & 990 & 15,341 & 15,257 & 311 \\
\hline zinc_leads & - & - & - & - & - & - \\
\hline
\end{tabular}

The time taken by the new implementation includes adjacency conversion (Table 2). Unprocessed measurements are provided in Additional file 2: Short cycles.

basis if it is linearly independent from the current members of basis [27]. This check for linear independence is expensive $^{\mathrm{b}}$ and can be avoided under some conditions. With the union of all edges in the basis $\left(E_{B}\right)$ a new cycle is linearly independent if any edges of the candidate $\left(E_{\text {cand }}\right)$ are not present in basis. That is, when $\left|E_{B} \cap E_{\text {cand }}\right|<$ $\left|E_{\text {cand }}\right|$, the cycle must independent. Additionally we know the basis is complete when the number of cycles is equal to the circuit rank. As the biconnected components are processed separately, the circuit rank of the component is $|E|-|V|+1$.

\section{Essential and relevant cycles}

The new implementation computes the relevant cycles directly [26]. The MCB and essential cycles are then

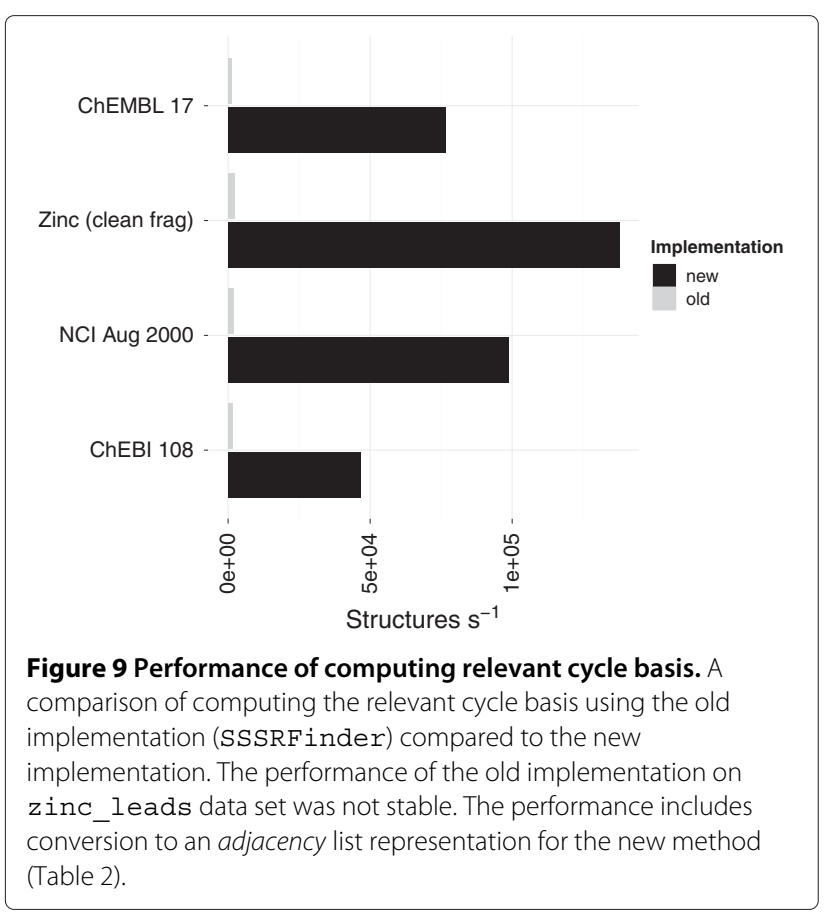

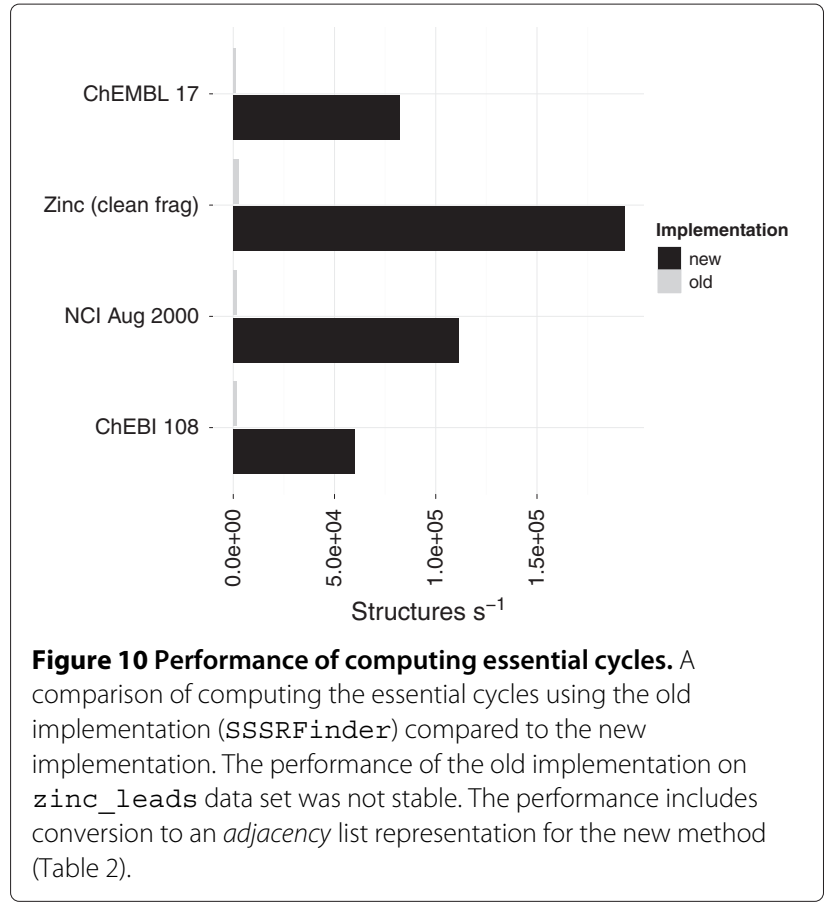

derived as a by-product. The computation of relevant and essential cycles showed a larger improvement over the existing implementations than for the MCB computation (Figures 9, 10). The old implementation could process around 2,000 structures per second whilst the newer implementations can process between 50,000 and 200,000 . The time taken to find all the relevant cycles in chembl_17 has dropped from $\sim 16$ minutes to only $\sim 16$ seconds (including 8 seconds spent in conversion) (Table 6). Similarly the time taken to find the essential cycles in the nci_aug0 0 dataset went from $\sim 2.5$ minutes to $\sim 2$ seconds (Table 7 ).

Previously the time taken to find the uniquely defined cycle sets was much longer than the non-unique $\mathrm{MCB}$ (Figure 11). The new implementation now finds the

Table 6 Average and median $(n=15)$ time taken to compute the relevant cycles using the existing and improved implementations

\begin{tabular}{lllllllll}
\hline \multirow{2}{*}{ Chemical structure } & \multicolumn{3}{c}{ Relevant cycles (old) } & & \multicolumn{3}{c}{ Relevant cycles (new) } \\
\cline { 2 - 3 } & $\begin{array}{l}\text { mean } \\
\boldsymbol{t}(\mathbf{m s})\end{array}$ & $\begin{array}{l}\text { median } \\
\boldsymbol{t} \text { (ms) }\end{array}$ & & & & $\begin{array}{l}\text { mean } \\
\text { (ms) }\end{array}$ & $\begin{array}{l}\text { median } \\
\boldsymbol{t}(\mathbf{m s})\end{array}$ & sdev \\
\hline chebi_108 & 17,013 & 16,897 & 576 & & 445 & 388 & 171 \\
nci_aug00 & 149,210 & 148,231 & 12,190 & 2,250 & 2,187 & 195 \\
zinc_frag & 183,587 & 184,720 & 18,219 & 2,610 & 2,519 & 237 \\
chembl_17 & 972,493 & 972,605 & 1,197 & 16,003 & 15,991 & 201 \\
zinc_leads & - & - & - & - & - & -
\end{tabular}

The time taken by the new implementation includes adjacency conversion (Table 2). Unprocessed measurements are provided in Additional file 2: Short cycles. 
Table 7 Average and median $(n=15)$ time taken to compute the essential cycles using the existing and improved implementations

\begin{tabular}{|c|c|c|c|c|c|c|}
\hline \multirow{2}{*}{ Chemical structure } & \multicolumn{3}{|c|}{ Essential cycles (old) } & \multicolumn{3}{|c|}{ Essential cycles (new) } \\
\hline & $\begin{array}{l}\text { mean } \\
t(\mathrm{~ms})\end{array}$ & $\begin{array}{l}\text { median } \\
t(\mathrm{~ms})\end{array}$ & sdev & $\begin{array}{l}\text { mean } \\
t(\mathrm{~ms})\end{array}$ & $\begin{array}{l}\text { median } \\
t(\mathrm{~ms})\end{array}$ & sdev \\
\hline chebi_108 & 16,561 & 16,395 & 615 & 572 & 424 & 451 \\
\hline nci_aug00 & 128,963 & 128,663 & 2,325 & 2,536 & 2,459 & 362 \\
\hline zinc_frag & 217,312 & 217,016 & 940 & 3,662 & 3,574 & 336 \\
\hline chembl_17 & 954,954 & 952,437 & 20,698 & 17,235 & 17,171 & 293 \\
\hline zinc_leads & - & - & - & - & - & - \\
\hline
\end{tabular}

The time taken by the new implementation includes adjacency conversion (Table 2). Unprocessed measurements are provided in Additional file 2: Short cycles.

unique cycle sets in time comparable to the non-unique MCB (Figure 12).

The number of cycles found by each set was also measured on the data sets (Table 8). On average the number of cycles found in the unique sets was within $1 \%$ of the non-unique $\mathrm{MCB}$. This means in practice the unique sets can readily be used as a replacement for the non-unique MCB. It is however inevitable that some structures will have an infeasible number of relevant cycles. During testing, a structure in PubChem-Compound (CID 53389303) was found to contain over 1 million relevant cycles. As the number of relevant cycles can be determined without generating the walks, such structures can be filtered out if desired.

\section{All elementary cycles}

The data structures of AllRingsFinder were optimised and the timeout replaced with a threshold specific

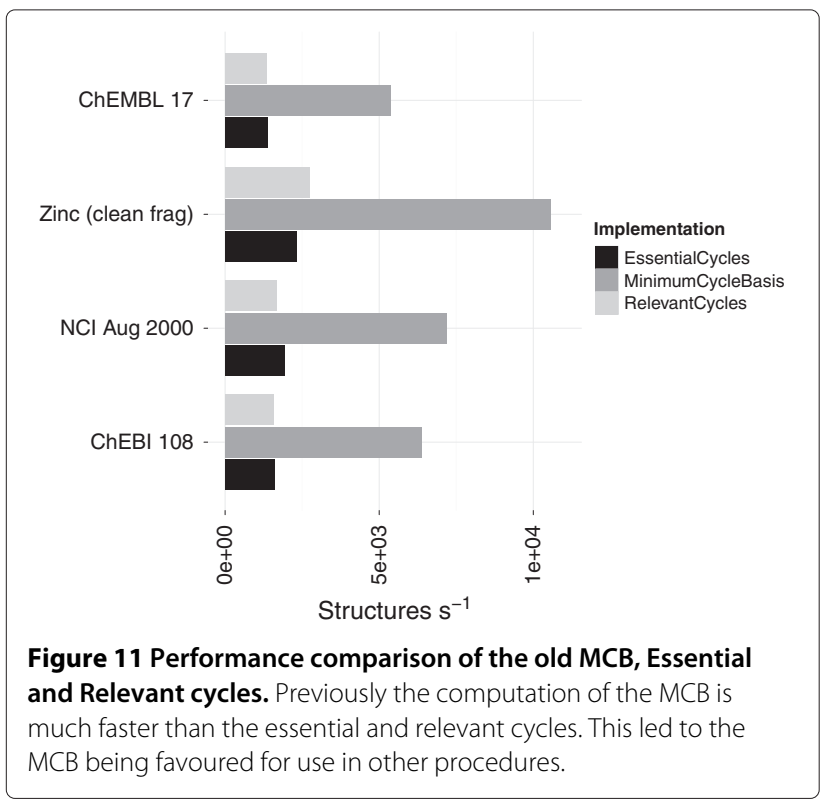

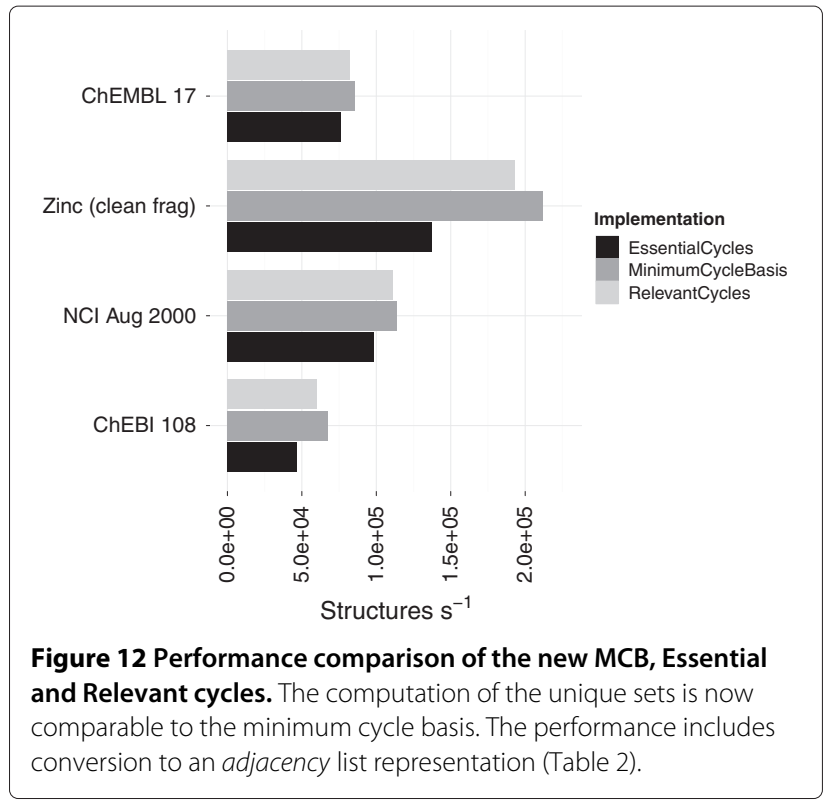

to the algorithm [20]. The improvements to the data structures involved representing the path-graph as an incidence-list and using binary sets to test intersection. The algorithm progresses by iteratively reducing (removing) vertices - the order of removal can be predetermined or dynamic. Using a predetermined order the edges need only be indexed by the next endpoint (i.e. directed). This reduces the number of modifications to the path-graph. Edges are only removed when a vertex is being reduced and all edges can be removed at once from this vertex. As each vertex is reduced the degree on the adjacent vertices may increased. Limiting the maximum degree the algorithm is allowed to reach provides a better threshold to determine feasibility.

To determine an appropriate threshold the algorithm was run on all fused ring systems found in PubChem Compound (Dec 2012). The maximum degree required to perceive the ring systems was measured for each system. An arbitrarily high value of $(d e g=5000)$ was chosen as an absolute maximum value. It was found that $987(0.005 \%)$ systems would require a threshold larger than maximum

\section{Table 8 The number of cycles in each set}

\begin{tabular}{llllll}
\hline $\begin{array}{l}\text { Chemical } \\
\text { structure }\end{array}$ & $\boldsymbol{n}$ structures & MCB & Essential & Relevant & All \\
\hline chebi_108 & 26,790 & 56,572 & 55,687 & 57,401 & $\sim 126,713$ \\
nci_aug00 & 250,172 & 599,876 & 591,144 & 606,045 & $\sim 1,007,643$ \\
zinc_frag & 504,074 & 880,296 & 875,801 & 882,393 & $\sim 1,022,498$ \\
chembl_17 & $1,318,180$ & $4,505,285$ & $4,455,907$ & $4,563,027$ & $\sim 6,599,942$ \\
zinc_leads & $5,135,179$ & - & - & - & $\sim 14,816,752$
\end{tabular}

The counts for all elementary cycles are approximate as it was infeasible to finish computation on some structures. 
Table 9 Ring systems (PubChem-Compound) that were feasibly handled by the improved AllRingsFinder at different thresholds

\begin{tabular}{llll}
\hline Percentile & Threshold (degree) & $\begin{array}{l}\text { Feasible } \\
\text { ring systems }\end{array}$ & $\begin{array}{l}\text { Infeasible } \\
\text { ring systems }\end{array}$ \\
\hline 99.95 & 72 & $17,834,013$ & 8,835 \\
99.96 & 84 & $17,835,876$ & 6,972 \\
99.97 & 126 & $17,837,692$ & 5,156 \\
99.98 & 216 & $17,839,293$ & 3,555 \\
99.99 & 684 (default) & $17,841,065$ & 1,783 \\
\hline 99.991 & 882 & $17,841,342$ & 1,506 \\
99.992 & 1,062 & $17,841,429$ & 1,419 \\
99.993 & 1,440 & $17,841,602$ & 1,246 \\
99.994 & 3,072 & $17,841,789$ & 1,059 \\
\hline 99.9946 & 5,000 (max tested) & $17,841,861$ & 987 \\
\hline
\end{tabular}

5000 to finish. Retrospectively calculating the threshold required for a given percentile showed only a small gain for higher values (Table 9). Even using a small threshold of only 9 allows perception of $99 \%$ of the ring systems (Figure 13). The default value (used in benchmarks) was chosen as 684 which from this test would allow the algorithm to feasibly complete on $\sim 99.99 \%$ of the systems present in PubChem Compound (Dec 2012).

The new threshold still encounters infeasible structures but number found is fewer and does not vary between runs (Table 10). The performance of the new implementation was compared against the old algorithm using a time out of $5 \mathrm{~ms}$ (much lower than default). With the small timeout 2,500 structures in chembl_17 were considered infeasible by the old implementation whilst using the new implementation with the default threshold only
232 are infeasible. The new algorithm was able to compute more cycles (Table 11) in less time (Figure 14). The time taken to find all cycles in chembl_17 previously took $\sim 9$ minutes (with a small threshold) but now takes only $\sim 25$ seconds (Table 12 ). Disregarding the conversion we found that when the computation was feasible, determining all cycles was as fast as the smaller subsets.

\section{Additional cycles sets}

The shortest cycle through each vertex and edge is also provided as a unique but potentially exponential cycle set. The edge-short cycles has also been termed the Largest Set of Smallest Rings (LSSR) and is utilised within Open Babel [28]. Computation of the sets does not check if the cycles form a basis. This could improve performance but no noticeable change was observed in measurements. The implementations are provided for compatibility.

A TripletCycles utility was also implemented to improve generation of CACTVS [29] Substructure Keys (PubChemFingerprint). These cycles are the shortest through a vertex triple $\{u, v, w\}$ and allows generation of cycles for envelope rings such as naphthalene or azulene whilst avoiding larger fused rings. The implementation allows a unique or non-unique set to be generated.

\section{Conclusion}

The improved performance in cycle perception means it is now feasible to analyse much larger chemical data sets. This is particularly true of the unique short cycle sets (essential and relevant) which saw an order of magnitude improvement. It is now no longer favourable to utilise the non-unique MCB due to runtime performance. Any procedures incorrectly relying on the MCB to be unique can

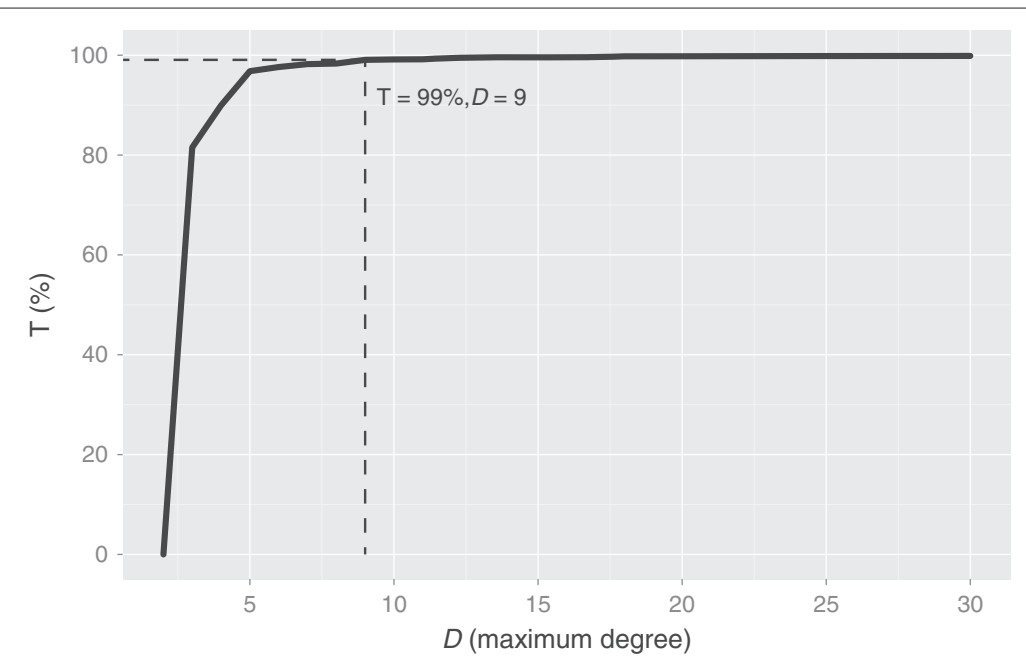

Figure 13 Degree threshold required to perceive percentage of fused ring systems. Percentage $(T)$ of feasible ring systems in PubChem Compound (Dec 2012) for a given degree (D). 
Table 10 Average $(n=15)$ number of structures considered infeasible by the old and new implementations

\begin{tabular}{llll}
\hline $\begin{array}{l}\text { Chemical } \\
\text { structure }\end{array}$ & $\boldsymbol{n}$ structures & $\boldsymbol{n}$ fail (old) & $\boldsymbol{n}$ fail (new) \\
\hline chebi_108 & 26,790 & $108-117$ & 41 \\
nci_aug00 & 250,172 & $306-311$ & 37 \\
zinc_frag & 504,074 & 0 & 0 \\
chembl_17 & $1,318,180$ & $2528-2547$ & 232 \\
zinc_leads & $5,135,179$ & 0 & 0
\end{tabular}

Based on a timeout procedure the number of cycles found in the old procedure varied between repeats, given the same structures the new feasibility threshold does not.

be easily adapted to use the new algorithms. The efficient implementation of the relevant cycles could also be adapted to compute a recent descriptor known as Unique Ring Families [30].

Improvements were seen throughout the toolkit with cycle perception being required for core functionality. The new algorithm for cycle membership has been used to improve performance of atom typing and the set of all cycles utilised in aromaticity perception. To avoid a performance hit from the old implementation the aromaticity of non-shortest cycles was only perceived for small fused rings systems. The new aromaticity has no restrictions and attempts to perceive aromaticity on all cycles. If computation is not feasible the aromaticity perception falls back to a smaller more feasible cycle set. Alternatively the smaller set of cycles could be tested first with the larger set only utilised if potentially aromatic atoms were remaining. Using the optimised representations the set of all cycles is generally faster to compute than the smaller sets and it is preferable to try all and fail fast.

A large portion of the time is spent in converting the CDK objects to optimised representations. Despite this without the conversion the runtime performance is much slower. Further gains could be made by optimising the native data structures and removing the need for conversion. The changes required would be large but could be introduced in future releases.

Table 11 Average $(n=15)$ number of all cycles found in each datasets

\begin{tabular}{llllllll}
\hline \multirow{2}{*}{ Chemical structure } & $\boldsymbol{n}$ structures & \multicolumn{2}{c}{ Old } & & \multicolumn{2}{c}{ New } \\
\cline { 3 - 4 } & & Cycles & sdev & & Cycles & sdev \\
\hline chebi_108 & 26,790 & 98,597 & 199 & 126,713 & 0 \\
nci_aug00 & 250,172 & 936,625 & 409 & $1,007,643$ & 0 \\
zinc_frag & 504,074 & $1,022,498$ & 0 & & $1,022,498$ & 0 \\
chembl_17 & $1,318,180$ & $6,176,585$ & 378 & $6,599,942$ & 0 \\
zinc_leads & $5,135,179$ & $14,816,752$ & 0 & & $14,816,752$ & 0
\end{tabular}

Due to the time out the old implementation sometimes provided different counts for each repeat.

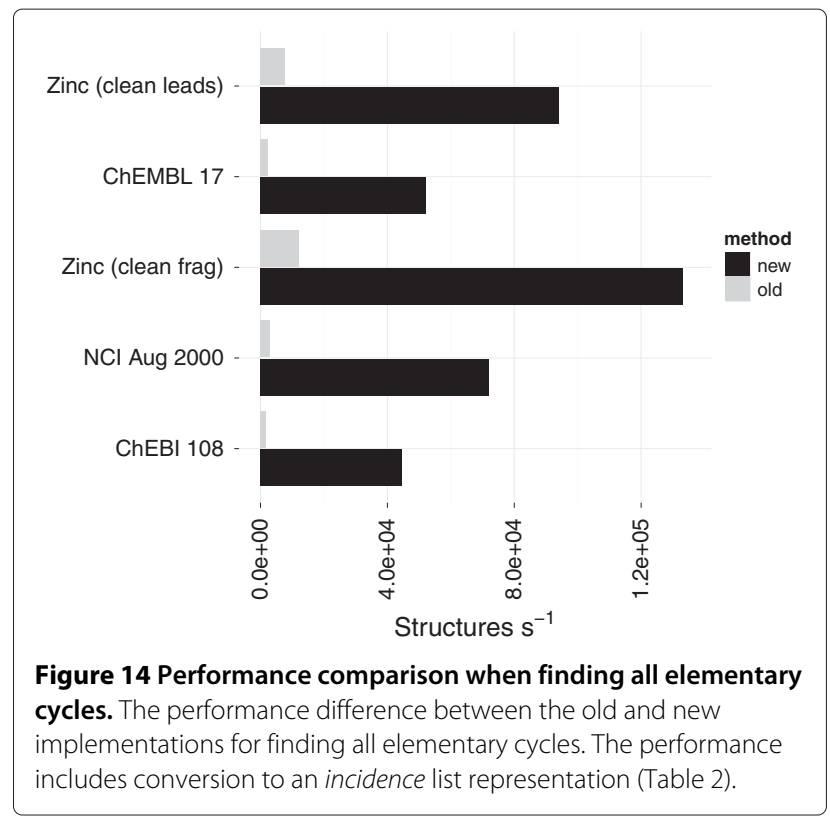

\section{Availability and requirements}

Project Name: The Chemistry Development Kit

Project Home Page: sourceforge.net/projects/cdk/ (version CDK (development)) or github.com/cdk/cdk (version 1.5.4 onwards)

Operating System: Platform Independent

Programming Language: Java

Requirements: Java 1.6+

License: Lesser General Public License 2.1

\section{Endnotes}

aalternatively known as cyclomatic number, nullity $(\mu)$, frère jacque number, first Betti's number or bond closures [5].

${ }^{b}$ linear independence is check with row reduction of a matrix (Gaussian elimination).

Table 12 Average and median $(n=15)$ time taken to find all rings using existing and improved implementations of AllRingsFinder

\begin{tabular}{|c|c|c|c|c|c|c|}
\hline \multirow{2}{*}{ Chemical structure } & \multicolumn{3}{|c|}{ Old } & \multicolumn{3}{|c|}{ New } \\
\hline & $t(\mathrm{~ms})$ & $\begin{array}{l}\text { median } \\
t \text { (ms) }\end{array}$ & sdev & $t(\mathrm{~ms})$ & $\begin{array}{l}\text { median } \\
t(\mathrm{~ms})\end{array}$ & sdev \\
\hline chebi_108 & 16,293 & 16,179 & 540 & 599 & 574 & 105 \\
\hline nci_aug00 & 80,417 & 80,339 & 319 & 3,478 & 3,449 & 143 \\
\hline zinc_frag & 41,854 & 41,747 & 458 & 3,786 & 3,778 & 60 \\
\hline chembl_17 & 568,984 & 568,809 & 829 & 25,200 & 25,181 & 99 \\
\hline zinc_leads & 661,028 & 661,368 & 1133 & 54,490 & 54,471 & 101 \\
\hline
\end{tabular}

The newer code includes the overhead of conversion to (and from) the CDK objects. (Table 2). Unprocessed measurements are provided in Additional file 3: All cycles. 


\section{Additional files}

Additional files 1: Cycle membership benchmark. Performance measurements of determining cycle membership using SpanningTree and Ringsearch

Additional files 2: Short cycles. Performance measurements of determining short cycles (MCB, essential and relevant) using the old and new implementations.

Additional files 3: All cycles. Performance measurements of determining all cycles using the old and new implementations.

\section{Abbreviations}

The following abbreviations have been used in reference to implementation class names in the CDK library. Here we detail the full package name used in library. AllRingsFinder: org.openscience.cdk.ringsearch.AllRingsFinder Cycles : org.openscience.cdk.graph.Cycles; MinimumCycleBasis : org openscience.cdk.graph.MinimumCycleBasis; PubChemFingerprint : org openscience.cdk.fingerprint.PubChemFingerprint; RingSearch: org. openscience.cdk.ringsearch.RingSearch; SSSRF inder : org.openscience. cdk.ringsearch.SSSRFinder; SpanningTree : org.openscience.cdk.graph SpanningTree; TripletCycles : org.openscience.cdk.graph.TripletCycles.

\section{Competing interests}

The authors declare that they have no competing interests.

\section{Authors' contributions}

JM implemented, benchmarked the ring perception algorithms and wrote the manuscript. CS designed the core library and provides continuous support. Both authors read and approved the final manuscript.

\section{Acknowledgements}

This work was supported by Biotechnology and Biological Sciences Research Council CASE studentship [BB/1532153/1]. The authors would like to thank the following persons for their valuable discussions (names are alphabetically ordered): Felicity Allen, Stephan Beisken, Gordon James, Luis de Figueiredo, Pablo Moreno and Kalai Vannai. Additionally we would like to specifically thank Egon Willighagen for his thorough review of CDK additions.

Received: 17 December 2013 Accepted: 27 January 2014

Published: 30 January 2014

References

1. Steinbeck C, Han Y, Kuhn S, Horlacher O, Luttmann E, Willighagen E: The Chemistry Development Kit (CDK): an open-source java library for chemo- and bioinformatics. J Chem Inf Comput Sci 2003, 43(2):493-500

2. Steinbeck C, Hoppe C, Kuhn S, Floris M, Guha R, Willighagen E: Recent developments of the Chemistry Development Kit (CDK) - an open-source java library for chemo- and bioinformatics. CPD 2006 , 12:2111-2120.

3. Berger F, Flamm C, Gleiss PM, Leydold J, Stadler PF: Counterexamples in chemical ring perception. $J$ Chem Inf Comput Sci 2004, 44(2):323-331.

4. Downs $G$, Gillet $V$, Holliday J, Lynch M: Review of ring perception algorithms for chemical graphs. J Chem Inf Comput Sci 1989, 29(3):172-187.

5. Downs G: Ring Perception, Handbook of Cheminformatics, vol 1. Weinheim: Wiley-VCH; 2003.

6. Willett P: Similarity-based virtual screening using 2D fingerprints. Drug Discovery Today 2006, 11(23):1046-1053.

7. Daylight: SMARTS - A Language for describing molecular patterns. http://www.daylight.com/dayhtml/doc/theory/theory.smarts.html.

8. Razinger M, Balasubramanian K, Perdih M, Munk M: Stereoisomer generation in computer-enhanced structure elucidation. J Chem Inf Comput Sci 1993, 33(6):812-825.

9. Balaban AT: Applications of graph theory in chemistry. J Chem Inf Comput Sci 1985, 25:334-343.

10. Bolton $E$, Wang $Y$, Thiessen $P$, Bryant S: PubChem: Integrated platform of small molecules and biological activities. In Chapter 12 in Annual Reports in Computational Chemistry, Volume 4. Washington: American Chemical Society; 2008.
11. Nikolova-Jeliazkova N: Slow fingerprints? CDKNews 2005, 2(2):34-40.

12. Kruskal JB: On the shortest spanning subtree of a graph and the traveling salesman problem. Proc Am Math Soc 1956, 7(1):48-50.

13. Figueras J: Ring perception using breadth-first search. $J$ Chem Inf Comput Sci 1996, 36:986-991.

14. RDKit: Cheminformatics and machine learning software 2014. http:// www.rdkit.org

15. Hastings J, Matos PD, Dekker A, Ennis M, Harsha B, Kale N, Muthukrishnan V, Owen G, Turner S, Williams M, Steinbeck C: The ChEBI reference database and ontology for biologically relevant chemistry: enhancements for 2013. Nucleic Acids Res 2013, 41(D1):456-463.

16. National Cancer Institute (NCI) Database Download. http://cactus.nci. nih.gov/download/nci/.

17. Irwin J, Sterling T, Mysinger M, Bolstad E, Coleman R: ZINC: A free tool to discover chemistry for biology. J Chem InfModel 2012, 52(7):1757-1768.

18. Gaulton A, Bellis LJ, Bento AP, Chambers J, Davies M, Hersey A, Light $Y$, Mcglinchey S, Michalovich D, Al-Lazikani B, Overington JP: ChEMBL: a large-scale bioactivity database for drug discovery. Nucleic Acids Res 2012, 40(D1):1100-1107.

19. Weininger D: SMILES, A chemical language and information system. 1. Introduction to methodology and encoding rules. $J$ Chem Inf Comput Sci 1988, 28(1):31-36.

20. Hanser $T$, Jauffret $P$, Kaufmann $G$ : A new algorithm for exhaustive ring perception in a molecular graph. J Chem Inf Comput Sci 1996, 36(6):1146-1152.

21. Rahman S, Bashton M, Holliday GL, Schrader R, Thornton JM: Small Molecule Subgraph Detector (SMSD) toolkit. J Cheminformatics 2009 1(1):12.

22. Sedgewick R, Wayne K: Graphs, Algorithms, 4th edn. Boston: Addison Wesley; 2011

23. Skiena SS: Minimum spanning tree, Graphs, The Algorithm Design Manual. New York: Springer; 1997.

24. Hopcroft J, Tarjan R: Efficient algorithms for graph manipulation. Commun ACM 1973, 16(6):372-378.

25. Schwerdtfeger $P$, Wirz L, Avery J: Program FULLERENE: a software package for constructing and analyzing structures of regular fullerenes. J Comput Chem 2013, 34(17):1508-1526.

26. Vismara P: Union of all the minimum cycle bases of a graph. Electr $J$ Comb 1997, 4:73-879.

27. Horton JD: A polynomial-time algorithm to find the shortest cycle basis of a graph. SIAM J Comput 1987, 16(2):358-366.

28. O'Boyle NM, Banck M, James CA, Morley C, Vandermeersch T, Hutchison GR: Open Babel: an open chemical toolbox. J Cheminformatics 2001 3(1):33.

29. Ihlenfeldt W, Takahashi Y, Abe H, Sasaki S: Computation and management of chemical properties in CACTVS: an extensible networked approach toward modularity and compatibility. $J$ Chem InfComput Sci 1994, 34(1):109-116.

30. Kolodzik A, Urbaczek S, Rarey M: Unique ring families: a chemically meaningful description of molecular ring topologies. $J$ Chem Inf Model 2012, 52(8):2013-2021.

doi:10.1186/1758-2946-6-3

Cite this article as: May and Steinbeck: Efficient ring perception for the Chemistry Development Kit. Journal of Cheminformatics 2014 6:3. 